УДК 531.36, 531.38

Кононов Ю. М., Василенко В.Ю.

1 доктор фізико-математичних наук, професор,

2 аспірант кафедри прикладної механіки і комп'ютерних технологій, Донецький національний університет імені Василя Стуса

\title{
ПРО СТІЙКІСТЬ ОБЕРТАННЯ НЕСИМЕТРИЧНОГО ТВЕРДОГО ТІЛА ЗІ ЗБУДЖКЕННЯМ В СЕРЕДОВИЩІ, ЩО ЧИНИТЬ ОПІР
}

У припущенні, що центр мас твердого тіла знаходиться на третій головній осі інерції твердого тіла, на основі критерію Льєнара-Шипара, записаного в іннорному вигляді, отримані у вигляді системи трьох нерівностей умови асимптотичної стійкості рівномірного обертання динамічно несиметричного твердого тіла 3 нерухомою точкою. Тверде тіло знаходиться під дією сил тяжіння, дисипативного моменту і постійного моменту в інерціальній і неірціальній системах відліку. Проведені дослідження несиметрії твердого тіла і дисипативного моменту, а також двух постійних моментів на умови стійкості рівномірного обертання твердого тіла. Узагальнено відомі результати для симетричного твердого тіла на випадок динамічної несиметрії твердого тіла і дисипативної несиметрії.

Ключові слова: динамічно несиметричне тверде тіло, середовище, що чинить опір, асимптотична стійкість

\section{Вступ}

У статті [1] розглянуто задачу про вплив дисипативного моменту, що моделює опір середовища, і постійного моменту, прикладеного до зовнішньої рамки безінерційного карданова підвісу, на стійкість стаціонарних рухів симетричного твердого тіла. У роботі [2] ця задача була узагальнена на випадок струнного підвісу і рівномірних обертань вовчка. Стаття [3] узагальнює результати роботи [1] на випадок двох постійних моментів в інерціальній і неінерційній системах координат. Вплив малої несиметрії твердого тіла на стійкість стаціонарних рухів тіла було оцінено в роботах [4-5]. У статті [6-8] узагальнена задача [1] на випадок рівномірного обертання несиметричного твердого тіла і отримані у вигляді системи трьох нерівностей умови асимптотичної стійкості обертання несиметричного твердого тіла. В роботі [9] і в даній статті узагальнюються результати роботи [1] на випадок двох постійних моментів в інерціальній і неінерційній системах координат, а також узагальнюються в разі рівномірного обертання твердого тіла результати статті [3] на випадок несиметричного твердого тіла і несиметричної диссипації. Умови асимптотичної стійкості отримані на основі критерію Льенара-Шіпара, записаного в іннорном вигляді [10].

\section{1. Постановка завдання}

Розглянемо важке динамічно несиметричне тверде тіло, яке обертається навколо нерухомої точки, в припущенні, що на нього діє дисипативний момент

$$
\vec{M}_{d} \doteq-D \vec{\omega}\left(\operatorname{diag}\left(D_{1}, D_{2}, D_{3}\right), D_{i}>0, i=\overline{1,3}\right)
$$

який моделює середовище, що чинить опір, і два постійних момента $\vec{M}_{q}=Q \vec{\kappa}$ та $\vec{M}_{p}=P \vec{\gamma}$. Момент $\vec{M}_{p}$ постійний у інерціальній системі відліку, а момент $\vec{M}_{q}$ - в неінер- 
ційній, тобто в пов'язаній з твердим тілом системі відліку. Будемо вважати, що момент $\vec{M}_{q}$ спрямований по третій головній осі інерції твердого тіла, а також, що на цій осі знаходиться центр мас твердого тіла. Тут $\vec{\omega}$ - кутова швидкість твердого тіла, $\vec{k}$ - одиничний вектор третьої головної осі інерції твердого тіла, $\vec{\gamma}$ - одиничний вектор висхідної вертикалі, Р та $Q$ - довільна постійна. Рівняння руху твердого тіла в пов'язаної з ним системи координат мають вигляд [1-3]

$$
\begin{aligned}
J \bar{\omega}+\bar{\omega} \times(J \bar{\omega}) & =\bar{\gamma} \times \frac{\partial V}{\partial \gamma}+P \bar{\gamma}+Q \bar{k}-D \bar{\omega} \\
& \bar{\gamma}+\bar{\omega} \times \bar{\gamma}=0
\end{aligned}
$$

де $J=\operatorname{diag}\left(J_{1}, J_{2}, J_{3}\right)$ - тензор інерції твердого тіла для нерухомої точки, $V=\Gamma(\vec{k} \cdot \vec{\gamma})$ - потенційна енергія, $\Gamma=m g c, m$ - маса твердого тіла, $c$ - відстань від нерухомої точки до центру мас твердого тіла, $g$ - прискорення вільного падіння. Рівняння (1.1) виражає теорему про зміну кінетичного моменту $J \vec{\omega}$, а рівняння (1.2) - умова постійності вектору $\vec{\gamma}$ в інерціальній системі відліку. Проектуючи рівняння руху твердого тіла (1.1)-(1.2) на головній осі інерції твердого тіла для нерухомої точки, отримаємо [9]

$$
\begin{gathered}
J_{1} \omega_{1}+\left(J_{3}-J_{2}\right) \omega_{2} \omega_{3}=\Gamma \gamma_{2}-D_{1} \omega_{1}, \\
J_{2} \omega_{2}+\left(J_{1}-J_{3}\right) \omega_{3} \omega_{1}=-\Gamma \gamma_{1}-D_{2} \omega_{2}, \\
J_{3} \omega_{3}+\left(J_{2}-J_{1}\right) \omega_{1} \omega_{2}=P \gamma_{3}-D_{3} \omega_{3}, \\
\gamma_{1}+\omega_{2} \gamma_{3}-\omega_{3} \gamma_{2}=0 \\
\gamma_{2}+\omega_{3} \gamma_{1}-\omega_{1} \gamma_{3}=0 \\
\gamma_{3}+\omega_{1} \gamma_{2}-\omega_{2} \gamma_{1}=0 .
\end{gathered}
$$

Система (1.3)-(1.4) допускає рішення

$$
\begin{gathered}
\gamma_{1}=\gamma_{2}=0, \gamma_{3}=1, \omega_{1}=\omega_{2}=0, \omega_{3}=\omega=\frac{P+Q}{D_{3}}, \\
\gamma_{1}=\gamma_{2}=0, \gamma_{3}=-1, \omega_{1}=\omega_{2}=0, \omega_{3}=\omega=\frac{-P+Q}{D_{3}},
\end{gathered}
$$

що відповідають рівномірним обертанням твердого тіла з кутовою швидкістю $\omega$ навколо вертикально розташованої третьої головної осі. При цьому рішенню (1.5) відповідає випадок "сплячого"вовчка"(центр мас твердого тіла знаходиться вище нерухомої точки, тобто $c>0)$, на який діє перекидний момент $(\Gamma>0)$, моменти $\vec{M}_{q}$ та $\vec{M}_{p}$, а рішенню (1.6) - випадок статично стійкого "вовчка"(центр мас знаходиться нижче нерухомої точки $(c<0)$,на який діє момент, що відновлюється $(\Gamma<0)$ та моменти $\vec{M}_{q}-\vec{M}_{p}$.

2. Асимптотична стійкість рішень (1.5) - (1.6).

Вважаючи в обуреному русі $\gamma_{3}=1+\delta, \omega_{3}=\omega+\sigma$ і зберігаючи для інших змінних їх колишні позначення, запишемо лінеаризовані рівняння обуреного руху

$$
\begin{gathered}
J_{1} \omega_{1}+\left(J_{3}-J_{2}\right) \omega_{2} \omega-\Gamma \gamma_{2}-P \gamma_{2}+D_{1} \omega_{1}=0 \\
J_{2} \omega_{2}+\left(J_{1}-J_{3}\right) \omega_{1} \omega+\Gamma \gamma_{1}-P \gamma_{2}+D_{2} \omega_{2}=0 \\
J_{3} \sigma+D_{3} \sigma-P \sigma=0 \\
\gamma_{1}+\omega_{2}-\omega \gamma_{2}=0 \\
\gamma_{2}-\omega_{1}+\omega \gamma_{1}=0 \\
\delta=0
\end{gathered}
$$

Кононов Ю. М., Василенко В.Ю. 
При динамічній $\left(J_{2}=J_{1}\right)$ і дисипативній $\left(D_{2}=D_{1}\right)$ симетрії рівняння $(2.1)$ збігаються 3 рівняннями роботи [3], а рівняння (2.2) залишаються без змін. У зв'язку з цим всі основні властивості рівнянь [3] переносяться і на рівняння (2.1) - (2.2), а саме:характеристичне рівняння системи (2.1)-(2.2) завжди має один нульовий корінь, обумовлений наявністю геометричного інтеграла $\gamma \frac{2}{1}+\gamma \frac{2}{2}+\gamma \frac{2}{3}=1$, і один негативний корінь $-D_{3} / J_{3}$; перше і друге рівняння системи (2.1) і відповідно системи (2.2) відокремлюються від інших рівнянь, i рішення (1.5)-(1.6) асимптотично стійкі, якщо всі корені характеристичного рівняння цих рівнянь мають негативні речові частини, і нестійкий, якщо хоча б один корінь має позитивну речову частину. Асимптотична стійкість по змінній $\gamma_{3}$ випливає з асимптотичною стійкості на змінній $\gamma_{1}, \gamma_{2}$ і геометричного інтеграла. Для виведення характеристичного рівняння системи звичайних диференціальних рівнянь (2.1)-(2.2), як і в роботі [3], з перших двох рівнянь системи (2.2) висловимо $\omega_{1}, \omega_{2}$ і підставимо їх і їх похідні в перші два рівняння (2.1):

$$
\begin{aligned}
& J_{2} \ddot{\gamma}_{1}+D_{2} \dot{\gamma}_{1}+\Gamma_{1} \gamma_{1}-J_{s} \dot{\gamma}_{2}-\widetilde{D}_{2} \gamma_{2}=0 \\
& J_{1} \ddot{\gamma}_{2}+D_{2} \dot{\gamma}_{2}+\Gamma_{2} \gamma_{2}+J_{s} \dot{\gamma}_{1}+\widetilde{D}_{1} \gamma_{1}=0
\end{aligned}
$$

Тут

$$
\begin{gathered}
\Gamma_{1}=\left(J_{3}-J_{1}\right) \omega^{2}-\Gamma, \Gamma_{2}=\left(J_{3}-J_{2}\right) \omega^{2}-\Gamma, \\
J_{s}=J \omega, J=J_{1}+J_{2}-J_{3}>0, \widetilde{D}_{i}=D_{i} \omega-P(i=1,2) .
\end{gathered}
$$

Рівняння (2.3) описують рух лінійної механічної системи з двома ступенями свободи, що знаходиться під дією сил довільної структури: дисипативних, потенційних, гіроскопічних і циркуляційних. Останні також називаються силами радіальної корекції або непотенційними позиційними силами [1]. Основна відмінність отриманих рівнянь (2.3) від аналогічних рівнянь роботи [1] полягає в тому, що через динамічну $\left(J_{2} \neq J_{1}\right)$ і дисипативну $\left(D_{2} \neq D_{1}\right)$ несиметрію неможливо спростити характеристичне рівняння для системи (2.3), введенням комплексної функції $\gamma_{1}+i \gamma_{2}$. Характеристичне рівняння для системи (2.3) має вигляд

$$
\left(\lambda^{2} J_{1}+D_{1} \lambda+\Gamma_{2}\right)\left(\lambda^{2} J_{2}+D_{2} \lambda+\Gamma_{1}\right)+\left(J_{s} \lambda+\widetilde{D}_{1}\right)\left(J_{s} \lambda+\widetilde{D}_{2}\right)=0
$$

або

$$
a_{4} \lambda^{4}+a_{3} \lambda^{3}+a_{2} \lambda^{2}+a_{1} \lambda+a_{0}=0
$$

де

$$
\begin{gathered}
a_{4}=J_{1} J_{2}>0, a_{3}=J_{1} D_{1}+J_{2} D_{2}>0, \\
a_{2}=J_{s}^{2}+J_{1} \Gamma_{1}+J_{2} \Gamma_{2}+D_{1} D_{2}= \\
\left(2 J_{1} J_{2}-J_{3} J\right) \omega^{2}-\left(J_{1}+J_{2}\right) \Gamma+D_{1} D_{2}, \\
a_{1}=\left(\widetilde{D}_{1}+\widetilde{D}_{2}\right) J_{s}+D_{1} \Gamma_{1}+D_{2} \Gamma_{2}= \\
=\left(J_{1} D_{2}+J_{2} D_{1}\right) \omega^{2}-\left(D_{1}+D_{2}\right) \Gamma-2 J P \omega, \\
a_{0}=\Gamma_{1} \Gamma_{2}+\widetilde{D}_{1} \widetilde{D}_{2}=\left(J_{3}-J_{1}\right)\left(J_{3}-J_{2}\right) \omega^{4}+ \\
+\left[\left(J-J_{3}\right) \Gamma+D_{1} D_{2}\right] \omega^{2}-\left(D_{1}+D_{2}\right) P \omega+P^{2}+\Gamma^{2} .
\end{gathered}
$$

На підставі критерію Льенара - Шипара, записаного в іннорном вигляді (см. с.34 [7]), вивливає, що для того, щоб всі нулі рівняння (2.4) лежали у відкритій лівій півплощині необхідно і достатньо, щоб: 1) були позитивні все коефіцієнти (або половина цих коефіцієнтів); 2) були іннорно - позитивними матриці $\Delta_{3}^{e}$ та $\Delta_{1}^{e}$, тобто 


$$
I_{3}=\left|\Delta_{3}^{e}\right|=\left|\begin{array}{ccc}
a_{4} & a_{2} & a_{0} \\
0 & a_{3} & a_{1} \\
a_{3} & a_{1} & 0
\end{array}\right|=\left(a_{2} a_{3}-a_{1} a_{4}\right) a_{1}-a_{0} a_{3}^{2}>0,
$$

Таким чином, умови асимптотичної стійкості рішень (1.5), тобто коли діє перекидний момент $(\Gamma>0)$ має вигляд

$$
a_{0}>0, a_{1}>0 \text { i } I_{3}>0
$$

чи

$$
\begin{gathered}
\Gamma^{2}+\left(J-J_{3}\right) w^{2} \Gamma+\left[\left(J_{3}-J_{1}\right)\left(J_{3}-J_{2}\right) w^{2}+D_{1} D_{2}\right] w^{2}-\left(D_{1}+D_{2}\right) P w+P^{2}>0 \\
\left(D_{1}+D_{2}\right) \Gamma+2 J P w-\left(J_{1} D_{2}+J_{2} D_{1}\right) w^{2}<0 \\
\left(J_{1}-J_{2}\right)^{2} \Gamma^{2}+b_{1} \Gamma+b_{0}>0 .
\end{gathered}
$$

Тут

$$
\begin{gathered}
b_{1}=\left[J_{2}\left(J_{3}-2 J_{2}\right) D_{1}^{2}+\left(J_{1} J_{3}+J_{2} J_{3}-4 J_{1} J_{2}\right) D_{1} D_{2}+J_{1}\left(J_{3}-2 J_{1}\right) D_{2}^{2}\right] J w^{2}- \\
-\left(D_{1}+D_{2}\right)\left(J_{1} D_{1}+J_{2} D_{2}\right)+2\left(J_{1}-J_{2}\right)\left(J_{1} D_{2}-J_{2} D_{1}\right) J P w \\
b_{0}=-P\left[\left(J_{1} D_{2}+J_{2} D_{1}\right)^{2}+4 J_{1} J_{2} J^{2} w^{2}\right]- \\
-2 J_{3} J^{2}\left(J_{1} D_{1}+J_{2} D_{2}\right) w^{3}+\left(J_{1} D_{1}+J_{2} D_{2}\right)\left[D_{1} D_{2} \widehat{J}-J_{1} D_{2}^{2}-J_{2} D_{1}^{2}\right] w .
\end{gathered}
$$

Нерівність (2.6) можна представити в такий спосіб

$$
\left(\Gamma-\left(J_{3}-J_{1} w^{2}\right)\left(\Gamma-\left(J_{3}-J_{2} w^{2}\right)+\left(D_{1} w-P\right)\left(D_{2} w-P\right)>0,\right.\right.
$$

Умови асимптотичної стійкості рішення (1.6), тобто коли діє момент, який відновлюється $(\Gamma<0)$, слідують 3 нерівностей $(2.6)$ - (2.8) і коефіцієнтів $(2.9)$ в яких треба замінити $-P$ на $-P$ і вважати $w=\frac{-P+Q}{D_{3}}$ :

$$
\begin{gathered}
\Gamma^{2}+\left(J-J_{3}\right) w^{2} \Gamma+\left[\left(J_{3}-J_{1}\right)\left(J_{3}-J_{2}\right) w^{2}+D_{1} D_{2}\right] w^{2}+\left(D_{1}+D_{2}\right) P w+P^{2}>0 \\
\left(D_{1}+D_{2}\right) \Gamma-2 J P w-\left(J_{1} D_{2}+J_{2} D_{1}\right) w^{2}<0, \\
\left(J_{1}-J_{2}\right)^{2} \Gamma^{2}+\widetilde{b_{1}} \Gamma+\widetilde{b_{0}}>0 .
\end{gathered}
$$

Тут

$$
\begin{aligned}
& \widetilde{b_{1}}=\left[J_{2}\left(J_{3}-2 J_{2}\right) D_{1}^{2}+\left(J_{1} J_{3}+J_{2} J_{3}-4 J_{1} J_{2}\right) D_{1} D_{2}+J_{1}\left(J_{3}-2 J_{1}\right) D_{2}^{2}\right] J w^{2}- \\
& -\left(D_{1}+D_{2}\right)\left(J_{1} D_{1}+J_{2} D_{2}\right)+2\left(J_{1}-J_{2}\right)\left(J_{1} D_{2}-J_{2} D_{1}\right) J P w, \\
& \widetilde{b_{0}}=-P\left[\left(J_{1} D_{2}+J_{2} D_{1}\right)^{2}+4 J_{1} J_{2} J^{2} w^{2}\right]-2 J_{3} J^{2}\left(J_{1} D_{1}+J_{2} D_{2}\right) w^{3}+ \\
& +\left(J_{1} D_{1}+J_{2} D_{2}\right)\left[D_{1} D_{2} \widehat{J}-J_{1} D_{2}^{2}-J_{2} D_{1}^{2}\right] w .
\end{aligned}
$$

Кононов Ю. М., Василенко В.Ю. 


\section{3. Дослідження умов стійкості рішень (1.5)-(1.6).}

3 нерівності (2.12) випливає, що при дії моменту, що перекидаються $(\Gamma<0)$ воно буде завжди виконано при умові $P(Q-P) \geqslant 0$, тобто при $\mathrm{Q}>\mathrm{P}>\mathrm{Q}$ або $\mathrm{Q}<\mathrm{P}<\mathrm{Q}$. Таким чином, необхідні і достатні умови асимптотичної стійкості рівномірного обертання несиметричного твердого тіла зі збудженням в середовищі, що чинить опір при дії перекидаючого моменту $(\Gamma>0)$ записуються у вигляді трьох нерівностей (2.6) - (2.8), а при дії відновлюючого моменту $(\Gamma<0)$ - у вигляді трьох нерівностей $(2.11)-(2.13)$. Якщо $Q>P>O$ або $Q<P<O$, то $з$ цих трьох нерівностей залишаються дві нерівності (2.11) і (2.13).

Нерівності (2.6)-(2.8) і (2.11)-(2.13) щодо $w$ мають відповідно четверту, другу і третю ступенів. 3 нерівностей (2.6)-(2.8) випливає, що при $\left(J_{3}-J_{1}\right)\left(J_{3}-J_{2}\right)>0, P(P+Q)>0$, i достатньо великих значеннях $|P+Q|$ ці нерівності будуть виконані, тому що відповідні коефіцієнти при старших ступенях в цих нерівностях позитивні. 3 нерівностей (2.11) (2.13) також випливає, що при $\left(J_{3}-J_{1}\right)\left(J_{3}-J_{2}\right)>0, P(P-Q)<0$, і досить великих значеннях $|P-Q|$ ці нерівності будуть виконані. Таким чином, якщо момент інерції $J_{3}$ $\epsilon$ найбілышим або найменшим моментом інерції, то при дії перекидаючого моменту $(\Gamma<0)$ і $P(P+Q)>0$, а також при дії відновлюючого моменту $(\Gamma<0)$ і $P(P-Q)<0$ рівномірне обертання несиметричного твердого тіла з порушенням в середовищі, що чинить опір, буде асимптотично стійко.

Досліджуємо стійкість положення рівноваги. Для цього в нерівностей (2.6)-(2.8) і (2.11)-(2.13) покладемо $w=0$. Для нерівностей (2.6)-(2.8) це означає $P=-Q$, а для (2.11)-(2.13) - $P=Q$. В цьому випадку нерівності (2.6) і (2.8) вірні, а (2.7) не виконано. Нерівності (2.11)-(2.13) всі виконані. Таким чином, тільки при дії відновлюючого моменту $(\Gamma<0)$ буде спостерігатися стійке положення рівноваги за умови рівності постійних моментів $(P=Q)$ або їх відсутності $(P=Q=0)$.

Розглянемо вплив малої динамічної і дисипативної несиметрії на умови стійкості (2.6)-(2.8) i (2.11)-(2.13). Для цього представимо $J_{2}$ i $D_{2}$ у вигляді

$$
J_{2}=J_{1}(1+\varepsilon), D_{2}=D_{1}\left(1+\varepsilon_{1}\right)
$$

де $|\varepsilon|=1,\left|\varepsilon_{1}\right|=1$.

Підставивши (3.1) в (2.7)-(2.8) і (2.10) $(\Gamma>0)$, з точністю до першого ступеня $\varepsilon$ i $\varepsilon_{1}$, отримаємо:

$$
\begin{gathered}
\left(\Gamma-\left(J_{3}-J_{1}\right) w^{2}\right)^{2}+\left(D_{1} w-P\right)^{2}+J_{1} w^{2}\left[\Gamma-\left(J_{3}-J_{1}\right) w^{2}\right] \varepsilon+ \\
+D_{1}\left(D_{1} w-P\right) w \varepsilon_{1}>0, \\
\Gamma<\frac{w}{2 D_{1}}\left[2 J_{1} D_{1} w+2\left(J_{3}-2 J_{1}\right) P+J_{1}\left(D_{1} W-2 P\right) \varepsilon-\left(J_{3}-2 J_{1}\right) P \varepsilon_{1}\right]>0, \\
b_{1} \Gamma+b_{0}>0 .
\end{gathered}
$$

Тут

$$
\begin{gathered}
b_{1}=-D_{1}^{2}\left(2\left[\left(J_{3}-2 J_{1}\right)^{2} w^{2}-D_{1}^{2}\right]+\left[\left(J_{3}-2 J_{1}\right)\left(J_{3}-6 J_{1}\right) w^{2}+D_{1}^{2}\right] \varepsilon+\right. \\
\left.+2\left[\left(J_{3}-2 J_{1}\right)^{2} w^{2}-2 D_{1}^{2}\right] \varepsilon_{1}\right) \\
b_{0}=2 P\left(2\left[J_{3}\left(J_{3}-2 J_{1}\right)^{2} D_{1} w^{3}-J_{1}\left(J_{3}-2 J_{1}\right)^{2} w^{2} P^{2}-J_{3} D_{1}^{3} w-J_{1} D_{1}^{2} P\right]+\right. \\
+\left[J_{3}\left(J_{3}-2 J_{1}\right)\left(J_{3}-6 J_{1}\right) D_{1} w^{3}-2 J_{1}\left(J_{3}-2 J_{1}\right)\left(J_{3}-4 J_{1}\right) w^{2} P-J_{3} D_{1}^{3} w-2 J_{1} D_{1}^{2} P\right] \varepsilon+ \\
\left.+\left[J_{3}\left(J_{3}-2 J_{1}\right)^{2} D_{1} w^{3}-3 J_{3} D_{1}^{3} w-2 J_{1} D_{1}^{2} P\right] \varepsilon_{1}\right)
\end{gathered}
$$


Підставивши (3.1) в (2.12)-(2.13) і (2.15) ( $Г<0)$, з точністю до першого ступеня $\varepsilon$ i $\varepsilon_{1}$, отримаємо:

$$
\begin{gathered}
\left(\Gamma-\left(J_{3}-J_{1}\right) w^{2}\right)^{2}+\left(D_{1} w-P\right)^{2}+J_{1} w^{2}\left[\Gamma-\left(J_{3}-J_{1}\right) w^{2}\right] \varepsilon+ \\
+D_{1}\left(D_{1} w-P\right) w \varepsilon_{1}>0, \\
\Gamma<\frac{w}{2 D_{1}}\left[2 J_{1} D_{1} w-2\left(J_{3}-2 J_{1}\right) P+J_{1}\left(D_{1} w+2 P\right) \varepsilon+\left(J_{3}-2 J_{1}\right) P \varepsilon_{1}\right]>0, \\
\widetilde{b_{1}} \Gamma+\widetilde{b_{0}}>0,
\end{gathered}
$$

де

$$
\begin{aligned}
& \widetilde{b_{1}}=-D_{1}^{2}\left(2\left[\left(J_{3}-2 J_{1}\right)^{2} w^{2}-D_{1}^{2}\right]+\left[\left(J_{3}-2 J_{1}\right)\left(J_{3}-6 J_{1}\right) w^{2}+\right.\right. \\
& \left.\left.+D_{1}^{2}\right] \varepsilon+2\left[\left(J_{3}-2 J_{1}\right)^{2} w^{2}-2 D_{1}^{2}\right] \varepsilon_{1}\right), \\
& \widetilde{b_{0}}=-2 P\left(2\left[J_{3}\left(J_{3}-2 J_{1}\right)^{2} D_{1} w^{3}-J_{1}\left(J_{3}-2 J_{1}\right)^{2} w^{2} P^{2}-J_{3} D_{1}^{3} w-J_{1} D_{1}^{2} P\right]+\right. \\
& +\left[J_{3}\left(J_{3}-2 J_{1}\right)\left(J_{3}-6 J_{1}\right) D_{1} w^{3}+\right. \\
& \left.+2 J_{1}\left(J_{3}-2 J_{1}\right)\left(J_{3}-4 J_{1}\right) w^{2} P-J_{3} D_{1}^{3} w+2 J_{1} D_{1}^{2} P\right] \varepsilon+ \\
& \left.+\left[J_{3}\left(J_{3}-2 J_{1}\right)^{2} D_{1} w^{3}-3 J_{3} D_{1}^{3} w+2 J_{1} D_{1}^{2} P\right] \varepsilon_{1}\right)
\end{aligned}
$$

3 нерівностей (3.2) та (3.7) випливає, що навіть маленька несиметрія може призвести до втрати стійкості.

Нерівність (3.2) буде виконана при

$$
\Gamma>\left(J_{3}-J_{1}\right) w^{2}, \varepsilon>0,\left[D_{1} Q-P\left(D_{3}-D_{1}\right)\right](Q+P) \geqslant 0, \varepsilon_{1}>0,
$$

а також при

$$
0<\Gamma<\left(J_{3}-J_{1}\right) w^{2}, \varepsilon>0,\left[D_{1} Q-P\left(D_{3}-D_{1}\right)\right](Q+P) \geqslant 0, \varepsilon_{1}>0 .
$$

Нерівність (3.7) буде виконана при

$$
0>\Gamma>\left(J_{3}-J_{1}\right) w^{2}, \varepsilon>0,\left[D_{1} Q+P\left(D_{3}-D_{1}\right)\right](Q-P) \geqslant 0, \varepsilon_{1}>0 .
$$

а також при

$$
\Gamma<\left(J_{3}-J_{1}\right) w^{2}, \varepsilon<0,\left[D_{1} Q+P\left(D_{3}-D_{1}\right)\right](Q-P) \leqslant 0, \varepsilon_{1}<0 .
$$

3 нерівностей (3.3) та (3.8) випливає, що маленька несиметрія може може зменшити запас стійкості при

$$
\left(J_{3}-2 J_{1}\right) P w \varepsilon_{1}>0,\left[D_{1} Q+\left(D_{1}-2 D_{3}\right) P\right] \varepsilon<0,(\Gamma>0)
$$

та

$$
\left(J_{3}-2 J_{1}\right) P w \varepsilon_{1}<0,\left[D_{1} Q-\left(D_{1}-2 D_{3}\right) P\right] \varepsilon<0,(\Gamma<0)
$$

- Дослідження проведені в рамках програми фундаментальних досліджень Міністерства освіти і науки України (проект № 0116U002522) і за грантової підтримки ДФФД (проект № $\Phi 71 / 47-2017)$.

Кононов Ю. М., Василенко В.Ю. 


\section{References}

[1] Kaрапетян A. В. Про вплив дисипативного і постійного моментів на вигляд і стійкість стаціонарних рухів волчка Лагранжа / А. В. Карапетян, І. С. Лагутіна // Изв. РАН. Механіка твердого тіла. - 1998. - №5. - С. 29-33.

[2] Kарапетлн A. В. Про стійкість рівномірних обертань волчка, підвішеного на струні, з урахуванням дисипативного і постійного мо-ментів / А. В. Карапетян, І. С. Лагутіна // Изв. РАН. Механіка твердого тіла. - 2000. - №1. - С. 53-57.

[3] Kарапетли A. В. Про стаціонарні рухи вовчка Лагранжа з порушенням в середовищі, що чинить опір / А. В. Карапетян // Вісник Московського ун-ту. Сер. 1. Математика. Механіка. - 2000. - №5. - С.39-43.

[4] Савченко А. Я. Стійкість руху систем пов'язаних твердих тіл / А. Я. Савченко, І. А. Болграбськая, Г.А.Кононихін. - К .: Наук. думка, 1991. - 166 с.

[5] Болграбсъкая I.A. Динаміка систем пов'язаних твердих тіл/ I. А. Болграбськая, М. Є. Лесіна, Д. А. Чебанов // Завдання та методи: математика, механіка, кібернетика. - Том 9. - К .: Наукова Думка, 2012. - 395 с.

[6] Кононов Ю.М. Вплив дисипативного і постійного моментів на стійкість рівномірного обертання твердого тіла / Ю.М. Кононов, Н.В. Кисельова, Д.В. Мішура // Вісник Донецького ун-ту. Сер.А. Природничі науки. - 2014. - №1. - С. 70-75.

[7] Kononov $Y u$. Stability of asymmetrical rigit body rotation with consideration of dissipative and constant moments / Yu. M. Kononov, V. Yu. Vasylenko, V. O. Proskuriakov // Book of Abstracts 5th INTERNATIONAL CONFERENCE of Young Scientists on Differential Equations and Applications dedicated to Yaroslav Lopatynsky, 9-11 November, 2016, Kyiv, Ukraine. - Vinnytsa 2016. -P. 84-86.

[8] Кононов Ю.М.Про стійкість обертання несиметричного твердого тіла з урахуванням диссипативного і постійного моментів / Ю. М. Кононов, В.Ю. Василенко // Вісн. Донецького ун-ту. Сер. А. Природничі науки - 2016.- № 1.

[9] Kononov Yu. The Stability of the Rigid Body Rotation with the Element of Excitation in the Resistant Environment / Yu. M. Kononov, V. Vasylinko // Book of Abstracts Iinternational Conference Differential Equations, Mathematical Physics, Cherkas, Ukraine. - Cherkas 2017.

[10] Джури Е. Іннори і стійкість динамічних систем / Е. Джури - М .: Наука, 1979. - 304 c.

Kononov Yu., Vasylenko V.

1 Doctor of Physical and Mathematical Sciences, Professor,

2 PhD student of the Department of Applied Mechanics and Computer Technologies, Vasyl' Stus Donetsk National University

\section{ON THE STABILITY OF ROTATION OF AN ASYMMETRIC SOLID BODY WITH EXCITATION IN A MEDIUM THAT RESISTS}

\section{SUMMARY}

Assuming that the center of mass of a solid is on the third principal axis of inertia of a rigid body, on the basis of the Lienard-Shipar criterion, written in an innocent form, the asymptotic stability condition for the uniform rotation of a dynamically asymmetric rigid body with a 
fixed point as a system of three inequalities. The solid is under the action of attraction forces, dissipative torque and constant moment in the inertial and non-reference frames. The carried out studies of the asymmetry of a solid body and the dissipative moment, as well as of two constant moments on the stability conditions for uniform rotation of a rigid body. The results for a symmetric rigid body in the case of dynamic asymmetry of a rigid body and dissipative asymmetry are known.

Key words: dynamically asymmetric solid, medium, resists, asymptotic stability.

\section{Кононов Ю.Н., Василенко В.Ю.}

1 доктор физико-математических наук, профессор,

2 аспирант кафедры прикладной механики и компютерных технологий, Донецкий начиональный университет имени Васыля Стуса

Об устойчивости вращения несимметричного твердого тела с возбуждением в среде, которая сопротивляется

\section{PЕЗЮМЕ}

В предположении, что центр масс твердого тела находится на третьей главной оси инерции твердого тела, на основе критерия Льенара-Шипара, записанного в иннорном виде, полученные в виде системы трех неравенств условия асимптотической устойчивости равномерного вращения динамически несимметричного твердого тела с неподвижной точкой. Твердое тело находится под действием сил притяжения, диссипативного момента и постоянного момента в инерциальной и неирциальний системах отсчета. Проведенные исследования несимметрии твердого тела и диссипативной момента, а также двух постоянных моментов на условия устойчивости равномерного вращения твердого тела. Обзор известны результаты для симметричного твердого тела в случае динамической несимметрии твердого тела и диссипативной несимметрии.

Ключевые слова: динамично несимметричное твердое тело, среда, сопротивляется, асимптотическая устойчивость. 